\title{
Módulo dinâmico de compósitos asfálticos com agregados sinterizados de argila calcinada
}

\section{(Dynamic modulus of asphalt mix with sintered calcined clay aggregate)}

\author{
C. L. da Silva, A. C. L. da Silva, C. A. da Frota \\ Grupo de Geotecnia, Faculdade de Tecnologia, Universidade Federal do Amazonas - UFAM, Av. General \\ Rodrigo Octávio Jordão Ramos 3000, Manaus, AM 69077-000 \\ cleudinei@gmail.com,cleiton.acls@hotmail.com,cafrota@ufam.edu.br
}

\begin{abstract}
Resumo
O presente estudo tem como principal objetivo a determinação do módulo dinâmico uniaxial, propiciando a obtenção de uma propriedade viscoelástica capaz de simular uma condição mais realística do que ocorre nos pavimentos, por força da demanda do tráfego. Trata-se de pesquisa inédita, com a participação do agregado sinterizado de argila calcinada (ASAC) em misturas asfálticas, visando a fomentar opção factível à pavimentação de vias urbanas no município de Manaus. Utilizaram-se, também, os seguintes materiais: seixo rolado, substituto regional à carência de material pétreo (referência), duas areias naturais, além de uma areia artificial proveniente da britagem de argila calcinada e o cimento asfáltico de petróleo 50/70 comercializado no estado do Amazonas. $\mathrm{Na}$ composição mineral das misturas, empregou-se o método Bailey, a enfatizar o intertravamento de seu esqueleto pétreo, a fim de se assegurar maior resistência à deformação permanente, principal patologia apresentada pelos pavimentos locais. Os resultados revelaram que, em altas temperaturas e em baixas frequências, as misturas com ASAC apresentaram-se mais rígidas relativas às misturas com seixo, indicando menos susceptibilidade às deformações permanentes, enquanto que, em baixas temperaturas e em altas frequências, o processo se inverteu e os compósitos com o agregado aluvionar passaram a expor valores de módulo dinâmico superiores aos das misturas com material alternativo. A avaliação mecânica, por meio do ensaio de Lottman, evidenciou que ambos os compósitos (com seixo e ASAC), apesar de apresentarem discretas perdas de resistência, preservaram suas estruturas, em consequência do efeito da umidade induzida. A inserção de areia artificial nas misturas asfálticas, a par de satisfatória quanto ao desempenho mecânico, minimiza a extração do recurso natural.
\end{abstract}

Palavras-chave: módulo dinâmico, viscoelasticidade, Lottman, agregado sinterizado de argila calcinada.

\begin{abstract}
The present study has as main objective the determination of uniaxial dynamic module, allowing obtaining viscoelastic properties able to simulate a more realistic condition that occurs in pavements, under the traffic demand. This is an original research, with the participation of the sintered calcined clay aggregate (SCCA) in asphalt mix, in order to promote feasible option for paving urban roads in the city of Manaus. We used also the following materials: river bed pebbles, substitute, given the lack of regional stone material (reference), two natural sands, plus artificial sand from the crushing plant calcined clay and asphalt cement 50/70 sold in the state of Amazonas. For mineral composition of the mixtures, we used the Bailey method, to emphasize the interlocking of their stony skeleton in order to ensure greater resistance to permanent deformation, primary pathology presented by local pavements. The results revealed that, at high temperatures and low frequencies, mixtures with SCCA were more rigid than those mixed with pebble, indicating less susceptibility to permanent deformation, while at low temperatures and at high frequencies, the process is reversed and composites with added alluvial began to expose dynamic modulus values higher than those of mixtures with alternative material. The mechanical evaluation by means of the Lottman assay, showed that both composites (with pebble and SCCA) techniques, although with slight loss of resistance, preserved their structures, due to the effect of moisture induced. The insertion of artificial sand in asphalt mix, beyond satisfactory on the mechanical performance, minimizes natural resource extraction.
\end{abstract}

Keywords: dynamic module, viscoelasticity, Lottman, sintered calcined clay aggregate.

\section{INTRODUÇÃO}

O município de Manaus encerra população cujo contingente se aproxima, a passos céleres, do patamar de dois milhões de habitantes, circunstância que torna a capital do estado do Amazonas a sétima cidade mais populosa do Brasil e a metrópole mais populosa da região Norte. A exemplo da parcela majoritária da região amazônica, Manaus se evidencia deficitária em jazidas de material pétreo superficial (brita), característica a encarecer o custo das obras de construção civil, em virtude das elevadas despesas com o transporte de tal matéria-prima, muitas vezes situada em afloramentos a grandes distâncias da área urbana manauense. Em consequência dessa conjuntura, as estruturas dos pavimentos de Manaus, em que trafega uma frota de 500 mil veículos [1] são, em regra, constituídas de material argiloso (subleito, sub-base e base), assim como de revestimento imbuído de mistura asfáltica do tipo 
AAUQ (areia asfalto usinada a quente), do que resulta a falência estrutural precoce, sobretudo em face do acúmulo excessivo de deformações permanentes na camada superficial, ocasionadas, principalmente, pela ausência de agregados graúdos nessas misturas. As misturas asfálticas, em decorrência do ligante (asfáltico) betuminoso presente em sua composição, possuem comportamento mecânico dependente de vários fatores, tais como a temperatura e a taxa de aplicação de carga ou deslocamento. A baixa temperatura e a alta taxa de carregamento, por exemplo, podem variar de elástico a viscoelástico linear, ao passo que a alta temperatura e a alta taxa de carregamento podem variar de viscoelástico não linear a viscoplástico [2]. Na determinação das propriedades viscoelásticas, vários ensaios podem ser realizados, tais como o módulo de relaxação, o creep estático (ou fluência) e o módulo dinâmico. No caso deste último, monitora-se a tensão ou a deformação no corpo de prova, relativamente a um carregamento senoidal, determinandose, então, a relação de amplitude do carregamento, por meio do deslocamento, bem como o tempo de defasagem entre os seus picos (ângulo de fase). $\mathrm{O}$ experimento pode ser realizado a várias frequências e a várias temperaturas, de sorte que, assim, são obtidos valores pontuais de módulo dinâmico, para cada par de frequência e temperatura. No domínio da frequência, as propriedades viscoelásticas, à luz dos processos matemáticos, podem, também, ser convertidas, com a finalidade de que se determinem as propriedades no domínio do tempo. Diante desse contexto, o presente estudo se dedica à caracterização viscoelástica linear de misturas asfálticas confeccionadas com agregados sinterizados de argila calcinada (ASAC), embasada no ensaio de módulo dinâmico - pesquisa almeja trazer a lume informações e resultados inéditos. Igualmente, o trabalho em pauta objetiva complementar pesquisas anteriores do Grupo de Geotecnia da Universidade Federal do Amazonas (GEOTEC/UFAM), direcionadas a aferir a viabilidade dos ASACs na confecção dos compósitos asfálticos, visando particularmente constituir-se em uma alternativa factível para suprir a carência de materiais pétreos naturais, em contraste com as limitações e fragilidades da tradicional pavimentação das vias urbanas manauaras [3-11]. Estudos com o citado material calcinado (ASAC) igualmente são levadas a efeito pelo Instituto Militar de Engenharia (IME), assim como pelo Instituto Alberto Luiz Coimbra de PósGraduação e Pesquisa de Engenharia da Universidade Federal do Rio de Janeiro (COPPE/UFRJ), as quais, de igual modo, indicam como promissor o uso desse agregado artificial [12-18].

\section{MATERIAIS E MÉTODOS}

Materiais: selecionou-se como matéria-prima para a confecção dos agregados sinterizados de argila calcinada (ASAC) um solo típico da Província Petrolífera de Urucu (SUC-N), a $650 \mathrm{~km}$ de Manaus. A obtenção dos ASACs consistiu na britagem de tijolos maciços $\left(60 \times 10 \times 200 \mathrm{~mm}^{3} \mathrm{de}\right.$ dois furos centrais de $\phi 16 \mathrm{~mm}$ ), produzidos especificamente para esse fim, utilizando-se as instalações de uma cerâmica tradicional do principal polo oleiro da região (Iranduba). Realizaram-se com a amostra natural (SUC-N) os seguintes experimentos: análise granulométrica [19], limite de liquidez [20] e limite de plasticidade [21]. Particularmente com os agregados sinterizados e antes de sua produção industrial, determinaram-se propriedades físicas - seleção expedita pelo processo de fervura [22], perda de massa após fervura [23] e desgaste por abrasão Los Angeles [24] - e químicas. Avaliaram-se os agregados graúdos quanto à granulometria [25], aos parâmetros Gsa, Gsb, Gsb ssd $_{\text {e absorção [26], as }}$ massas específica solta e compactada [27], ao desgaste pelo abrasão Los Angeles [28] e a adesividade ao ligante (asfáltico) betuminoso [29]. Escolheram-se três agregados miúdos para composição das misturas asfálticas: a) areia Mao, agregado usual da pavimentação regional; b) areia de Coari, empregada nos serviços de pavimentação do sistema viário da Província Petrolífera de Urucu; e c) areia de ASAC, resultante da britagem dos tijolos no processo de produção dos agregados sinterizados, que teve como finalidade proporcionar um melhor aproveitamento do material britado. Foram caracterizadas quanto à granulometria [25] e por meio da ASTM C 128 [30] determinaram-se as densidades (Gsa e Gsb) e absorção. Pela AASHTO T-19/T 19M-93 [31] calcularam-se as massas específicas compactadas. Utilizouse como ligante o cimento asfáltico de petróleo (CAP 50/70), distribuído pela refinaria de Manaus Isaac Sabbá (UN-Reman), tendo sido analisado pelas especificações da Superior Performance Asphalt Pavements - Superpave $[32,33]$. No caso do material fíler selecionou-se o cimento Portland, sendo caracterizado pela massa específica real [34] e granulometria [35].

Métodos: As composições granulométricas das misturas asfálticas seguiram a Faixa Superpave de Diâmetro Máximo Nominal (DMN) para 19,0 $\mathrm{mm}[32,33]$, tendo as porcentagens relativas dos agregados definidas pelo Método Bailey [35]. Determinou-se o teor de projeto pelo método do $3^{\circ}$ Distrito Rodoviário Federal ( $\left.3^{\circ} \mathrm{DRF}\right)$ do DNIT, que se baseia nos valores do Volume de Vazios (Vv) e na Relação Betume-Vazios (RBV), onde os limites máximos e mínimos são estabelecidos de acordo com a especificação do Método Marshall de dosagem [37]. Este igualmente se adotou na compactação dos corpos de prova. Tendo por fim simular o efeito de condicionamento de curto prazo, as misturas permaneceram, antes da compactação, $2 \mathrm{~h}$ em estufa, na temperatura $10^{\circ} \mathrm{C}$ superior à de compactação [38]. Fundamentado no Método de Ensaio DNER ME 004-94 [39] definiram-se as temperaturas de preparo e compactação das misturas e empregou-se o Rice Test [40] no cálculo da Densidade Específica Máxima (Gmm). A determinação experimental do módulo dinâmico $\left|E^{*}\right|$ ocorreu à tração com pulsos de cargas senoidais, representando a condição mais desfavorável do revestimento asfáltico. Realizouse este ensaio com uso de uma prensa multifuncional (Universal Testing Materials - UTM) IPC Global, equipada com mecanismos pneumáticos que possibilitam a aplicação 
de cargas dinâmicas, podendo-se variar tipos de pulsos, frequências e condições de confinamento. $\mathrm{O}$ citado equipamento também é dotado de diversos sensores para medição de carga, temperatura, pressão de confinamento e deslocamentos. Utilizou-se na suavização e regressão dos dados experimentais o software Origin, sendo os valores pontuais do módulo dinâmico $\left|E^{*}\right|$ e do ângulo de fase $\varphi$ calculados pelas seguintes equações A e B:

$$
\left|E^{*}\right|=\frac{\sigma 0}{\varepsilon 0}=\sqrt{\left(\mathrm{E}^{\prime}\right)^{2}+\left(\mathrm{E}^{\prime}\right)^{2}}
$$

na qual $E^{\prime}=\left|E^{*}\right| \cos \varphi, E^{\prime}=\left|E^{*}\right| \operatorname{sen} \varphi$ e $\varepsilon_{0}=$ deformação axial recuperável máxima

$$
\varphi=\frac{\mathrm{t}_{\mathrm{i}}}{\mathrm{t}_{\mathrm{p}}} 360
$$

na qual $t_{i}=$ fração de tempo entre os picos de tensão e deformação e $t_{p}=$ tempo de um ciclo de carga.

A partir desses valores pontuais de $\left|E^{*}\right|$, a várias frequências e temperaturas, construíram-se as curvas mestras, tendo-se, assim, os módulos dinâmicos a frequências muito elevadas ou baixas, em geral, não determinados experimentalmente por limitação dos equipamentos hoje disponíveis. Tais valores foram obtidos a partir dos fatores de translação horizontal $\left(\mathrm{a}_{\mathrm{T}}\right)$ calculados por meio da equação $\mathrm{C}$.

$$
\mathrm{a}_{\mathrm{T}}=\frac{\omega_{\text {Tref }}}{\omega}
$$

na qual $\omega_{\text {Tref }}$ e $\omega$ são as frequências relativas à temperatura de referencia e a temperatura em questão, respectivamente.

No ensaio Lottman utilizou-se também a UTM seguindo os procedimentos estabelecidos pela AASHTO T 283 [41]. Verificou-se a resistência de corpos-de-prova cilíndricos, com $63,5 \pm 1,3 \mathrm{~mm}$ de altura e diâmetro $101,5 \mathrm{~mm}$, por meio de ensaios à tração por compressão diametral, com as amostras submetidas aos condicionamentos previstos pelo referido método, a temperatura de $25 \pm 0,5^{\circ} \mathrm{C}$ com deformação controlada, e a uma taxa de deslocamento de 50 $\mathrm{mm} / \mathrm{min}$. A força de ruptura $(F)$ do $\mathrm{CP}$, necessária ao cálculo dos valores de RT, calculou-se pela seguinte equação D:

$$
\mathrm{RT}=\frac{2 \mathrm{~F}}{100 \pi \mathrm{DH}}
$$

na qual $[\mathrm{RT}]$ resistência à tração, em $\mathrm{MPa}, \mathrm{F}=$ carga de ruptura, em $\mathrm{N}, \mathrm{D}=$ diâmetro do corpo-de-prova, em $\mathrm{cm}$ e $\mathrm{H}=$ altura do corpo-de-prova, em $\mathrm{cm}$.

A razão de resistência à tração por ação da umidade induzida $(R R T)$ é dada por:

$$
\mathrm{RRT}=\frac{\mathrm{RT}_{\mathrm{c}}}{\mathrm{RT}_{\mathrm{nc}}}
$$

na qual $\mathrm{RT}_{\mathrm{nc}}=$ média da resistência à tração dos corposde-prova não condicionados; $\mathrm{RT}_{\mathrm{c}}=$ média da resistência à tração dos corpos-de-prova condicionados.

\section{RESULTADOS E DISCUSSÃO}

\section{Caracterização das amostras SUC-N e ASAC}

A Fig. 1 expõe a curva granulométrica referente à amostra no estado natural (SUC-N). Sua classificação textural apresenta as frações argila e silte [42], com um percentual de $92,0 \%$ de material passando na peneira com abertura da malha igual a $0,075 \mathrm{~mm}$, portanto atendendo a recomendação mínima de $85 \%$ estabelecida pelo DNIT para confecção de ASAC [5]. Os índices de consistência indicaram $\mathrm{LL}=48 \%$ e $\mathrm{LP}=22 \%$ ( $\mathrm{IP}=26 \%$ ). Segundo tais resultados o material se classifica como A7-6 (AASHTO/TRB) e CL (SUCS). Quanto aos resultados do material calcinado, indicados pelos ensaios de autoclave e de perda de massa (valor igual a 1,87\% [43]), o material ASAC mostrou-se aceitável para produção de agregados.

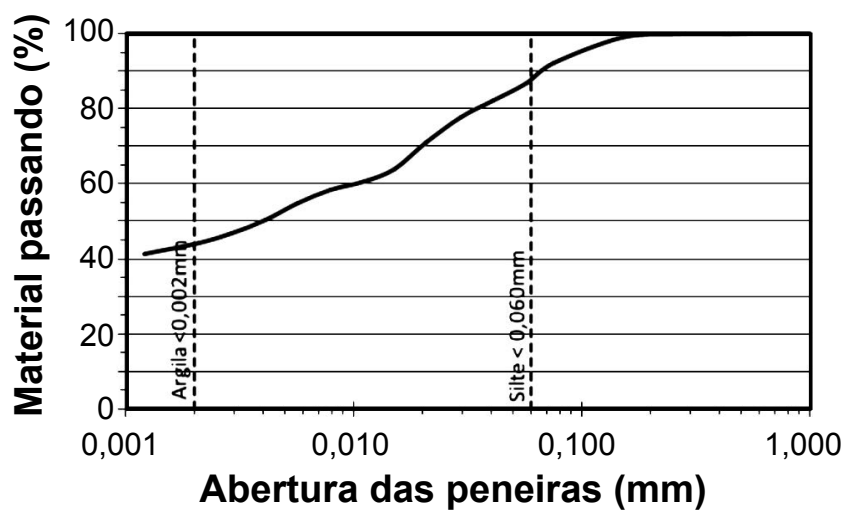

Figura 1: Curva granulométrica dos agregados graúdos. [Figure 1: Grain size distribution of the SUC-N sample.]

A caracterização química (Tabela I) das amostras no estado natural (SUC-N) e calcinada (ASAC) indicou que não se enquadraram inteiramente nos limites recomendados pelo DNIT e que a temperatura de queima não alterou a composição química. A análise mineralógica mostrou a presença dos minerais caulinita, goethita, hematita, ilita e quartzo.

Tabela I - Composição química [44].

[Table I - Chemical composition [44].]

\begin{tabular}{lccccc}
\hline Amostra & $\mathrm{SiO}_{2}$ & $\mathrm{Al}_{2} \mathrm{O}_{3}$ & $\mathrm{Fe}_{2} \mathrm{O}_{3}$ & $\mathrm{~K}_{2} \mathrm{O}$ & $\mathrm{TiO}_{2}$ \\
\hline SUC-N & 43,9 & 52,6 & 1,5 & 1,5 & 0,6 \\
ASAC & 46,7 & 50,1 & 1,5 & 1,1 & 0,6 \\
Recomendações & 50 a 65 & 15 a 20 & 5 a 10 & - & - \\
DNIT (1981) (\%) & & & & & \\
\hline
\end{tabular}

Caracterização dos agregados graúdos (ASAC e seixo)

Na Fig. 2 tem-se a curva granulométrica e na Tabela II os resultados dos ensaios de caracterização referentes aos agregados alternativos e naturais. Pela Fig. 2 e de acordo o que estabelece a ABNT NBR 6502 (1995), que tais materiais possuem predominância na fração granulométrica 


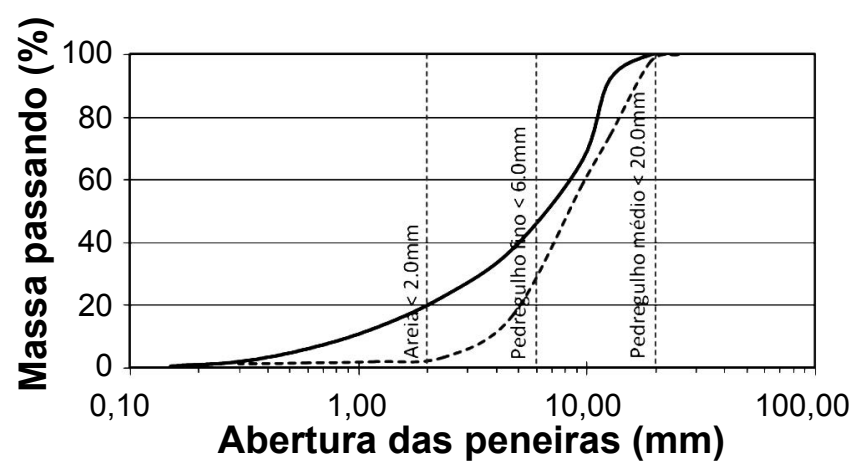

---- ASAC

Figura 2: Curva granulométrica dos agregados graúdos. [Figure 2: Grain size distribution of the coarse aggregates.]

Tabela II - Caracterização dos agregados graúdos. [Table II - Characterization of the coarse aggregates.]

\begin{tabular}{ccc}
\hline \multirow{2}{*}{ Análise } & \multicolumn{2}{c}{ Amostra } \\
& ASAC & Seixo \\
\hline $\mathrm{Gsb}\left(\mathrm{g} / \mathrm{cm}^{3}\right)$ & 1,855 & 2,626 \\
$\mathrm{Gsb} \mathrm{ssd}\left(\mathrm{g} / \mathrm{cm}^{3}\right)$ & 2,133 & 2,636 \\
$\mathrm{Gsa}\left(\mathrm{g} / \mathrm{cm}^{3}\right)$ & 2,571 & 2,643 \\
$\mathrm{Absorção}(\%)$ & 15,00 & 0,00 \\
Wul $\left(\mathrm{kg} / \mathrm{m}^{3}\right)$ & 1062,00 & 1894,60 \\
Wur $\left(\mathrm{kg} / \mathrm{m}^{3}\right)$ & 1126,35 & 1906,32 \\
Adesividade & Satisfatória & Satisfatória \\
\hline
\end{tabular}

entre 6,0 e $20,0 \mathrm{~mm}$. No caso do seixo, este apresenta aproximadamente $20 \%$ de sua textura passando na peneira \#2,00 mm (agregado miúdo), sendo o mesmo classificado como um pedregulho arenoso.

Nota-se pela Tabela II que: a) o ASAC, por se tratar de agregado leve, apresentou suas massas específicas Aparente, Solta e Compactada inferiores ao agregado aluvionar; b) o ASAC mostrou um alto potencial de absorção ( $15,00 \%)$; e c) a adesividade dos materiais foram satisfatórias, não havendo deslocamento da película do ligante, após as $72 \mathrm{~h}$ de ensaio. Os valores do ensaio de abrasão Los Angeles resultaram seixo $=37 \%$ e $\mathrm{ASAC}=70 \%$, onde: a) o material aluvionar atendeu as especificações estabelecidas (menor que 50\%); e b) o material alternativo mostrou um desgaste superior ao permitido. Apesar desse resultado, trabalhos [8, 9] demonstraram resistência mecânica apreciável de misturas asfálticas compostas com tais materiais quando comparadas aos compósitos com seixo.

\section{Caracterização dos agregados miúdos}

A Fig. 3 e a Tabela III indicam as curvas granulométricas (segundo a ABNT NBR 6502, 1995) e os resultados dos ensaios de caracterização alusivos aos agregados miúdos (Areia Mao, Areia Coari e Areia ASAC), respectivamente, onde se observa, que: a) areia de Manaus apresenta predominância média (fração entre 0,2 e $0,6 \mathrm{~mm}$ ); b) areia de Coari possui uma parcela média (aproximadamente $40 \%$ ), e mostra a fração grossa como predominante (cerca de $43 \%$ entre 0,6 e 2,0 mm); c) areia artificial (Areia ASAC) tem uma fração grossa (próximo de $37 \%$ ) e cerca de $35 \%$ retido na peneira \#2,00 $\mathrm{mm}$ (agregado graúdo), sendo classificada como uma areia pedregulhosa; e d) areia de ASAC, apontou suas massas específicas inferiores aos respectivos valores das areias convencionais (Mao e Coari).

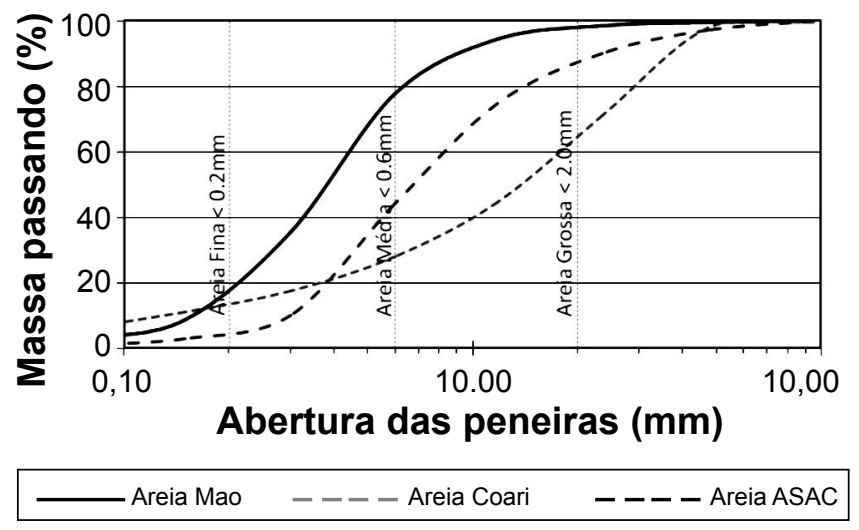

Figura 3: Curvas granulométricas dos agregados miúdos. [Figure 3: Grain size distribution of the fine aggregates.]

Tabela III - Caracterização dos agregados miúdos. [Table III - Characterization of the fine aggregates.]

\begin{tabular}{cccc}
\hline Amostra & Areia Mao & Areia Coari & Areia ASAC \\
\hline Gsb $\left(\mathrm{g} / \mathrm{cm}^{3}\right)$ & 2,632 & 2,627 & 1,790 \\
Gsb ssd $\left(\mathrm{g} / \mathrm{cm}^{3}\right)$ & 2,692 & 2,706 & 2,167 \\
Absorção $(\%)$ & 0,00 & 0,00 & 18,96 \\
Wur $\left(\mathrm{kg} / \mathrm{m}^{3}\right)$ & 1675,90 & 1782,20 & 1375,71 \\
\hline
\end{tabular}

\section{Caracterização CAP 50/70 e material Fíler}

Realizaram-se as análises do CAP 50/70, onde se nota pela Tabela IV que: a) todos os valores satisfazem aos parâmetros específicos; e b) com base nos valores alcançados e conforme critério Superpave, o citado ligante poderá ser utilizado em revestimentos asfálticos, na temperatura de serviço não superior a $64{ }^{\circ} \mathrm{C}$. No caso do cimento Portland, este passou integralmente na peneira de $\# 0,075 \mathrm{~mm}$, satisfazendo a especificação, conforme mostra a Tabela V. Sua massa específica real apontou o valor igual a $3,15 \mathrm{~g} / \mathrm{cm}^{3}$.

\section{Dosagens das misturas asfálticas}

$\mathrm{Na}$ Tabela VI tem-se as composições das misturas asfálticas do tipo CA determinadas com o método de Bailey. Concomitante a obtenção das curvas granulométricas, foram enquadradas na faixa Superpave com DMN de 19,0 mm, e segundo recomendado (Fig. 4) passando fora da Zona de Restrição (ZR) e entre os Pontos de Controle (PCs).

$\mathrm{O}$ teor de projeto da mistura com ASAC e seixo resultou 
Tabela IV - Características do cimento asfáltico de petróleo (CAP).

[Table IV - Characteristics of the petroleum asphalt cement.]

\begin{tabular}{|c|c|c|c|c|}
\hline \multicolumn{2}{|c|}{ Característica } & Unidade & Especificação & Resultado \\
\hline \multicolumn{2}{|c|}{ Ponto de Fulgor } & ${ }^{\circ} \mathrm{C}$ & 230 mín. & 301,0 \\
\hline \multirow{2}{*}{\multicolumn{2}{|c|}{$\begin{array}{c}\text { Viscosidade a } 135^{\circ} \mathrm{C} \\
\text { Perda de massa por envelhecimento } \\
\text { (RTFOT) }\end{array}$}} & $\mathrm{cP}$ & 3000 máx. & 382,5 \\
\hline & & $\%$ & 1,0 máx. & 0,4 \\
\hline \multicolumn{2}{|c|}{ Temperatura do ensaio de fadiga } & ${ }^{\circ} \mathrm{C}$ & $\mathrm{G}^{*} \operatorname{sen}(\delta)<5000 \mathrm{kPa}$ & 22,0 \\
\hline $\begin{array}{l}\text { Deformações } \\
\text { permanentes }\end{array}$ & $\begin{array}{l}\text { Antes do RTFO } \\
\text { Após o } \\
\text { RTFO }\end{array}$ & $\begin{array}{l}{ }^{\circ} \mathrm{C} \\
{ }^{\circ} \mathrm{C}\end{array}$ & $\begin{array}{l}\mathrm{G}^{*} / \operatorname{sen}(\delta)<1,00 \mathrm{kPa} \\
\mathrm{G}^{*} / \operatorname{sen}(\delta)<2,20 \mathrm{kPa}\end{array}$ & 64,0 \\
\hline
\end{tabular}

Tabela V - Especificação para material de enchimento. [Table $V$ - Specification of the filler.]

\begin{tabular}{ccc}
\hline \multirow{2}{*}{$\begin{array}{c}\text { Abertura da malha } \\
(\mathrm{mm})\end{array}$} & \multicolumn{2}{c}{ \% em peso, passando } \\
& Especificação & Cimento Portland \\
\hline 0,42 & 100 & 100 \\
0,18 & $95-100$ & 100 \\
0,075 & $65-100$ & 100 \\
\hline
\end{tabular}

Tabela VI - Composição das misturas CA.

[Table VI - Composition of the AC mixtures.]

\begin{tabular}{ccc}
\hline \multirow{2}{*}{ Componentes } & \multicolumn{2}{c}{ Mistura asfálticas } \\
& ASAC & Seixo \\
\hline ASAC & $74,25 \%$ & - \\
Seixo & - & $67,20 \%$ \\
Areia ASAC & $14,03 \%$ & - \\
Areia Coari & $10,32 \%$ & - \\
Areia Mao & - & $29,70 \%$ \\
Cimento Portland & $1,40 \%$ & $3,10 \%$ \\
\hline
\end{tabular}

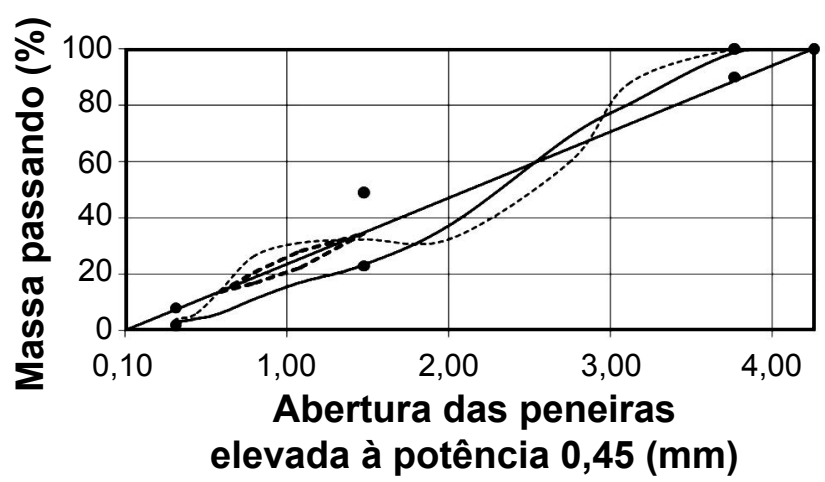

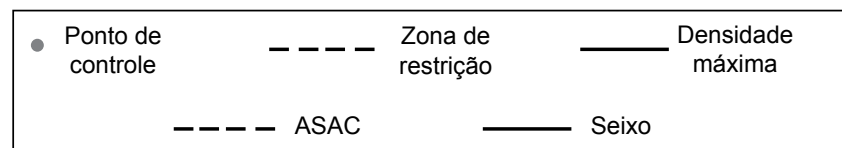

Figura 4: Curvas granulométricas das misturas com seixo e ASAC.

[Figure 4: Grain size distribution of the mixtures with pebble and SCCA.] no valor de $9,40 \%$ e $4,5 \%$, respectivamente. O maior valor para o compósito com o material alternativo mostra a influência do alto índice de absorção obtido para esses agregados.

As temperaturas de compactação, definidas pelo Método de Ensaio DNER ME 004-94, bem como as de preparo e envelhecimento em curto prazo $(2 \mathrm{~h})$, segundo a AASHTO PP-2, resultaram em, respectivamente: a) faixa de 148 a $157^{\circ} \mathrm{C}$; e b) faixa de 158 a $167^{\circ} \mathrm{C}, \log 010^{\circ} \mathrm{C}$ superiores à temperatura de compactação.

\section{Caracterização mecânica}

\section{Ensaio de umidade induzida (Lottman)}

Calculados os índices de vazios para todos os corpos de prova dos seis grupos estudados, moldados com compactador Marshall e segundo diferentes números de golpes por face (5, $15,25,35,45$ e 55 ), traçaram-se às curvas de compactação das misturas com seixo e com ASAC. A Fig. 5 apresenta tais curvas, com número de golpes por face $\times$ índice de vazios.

O número de golpes necessários para determinar o índice de vazios de $7 \%$ almejado para as misturas com seixo e com ASAC, calculados por meio das equações das curvas regredidas e visualizados na Fig. 5, foram, respectivamente, de 5 e 19 golpes.

Na Fig. 6 e Tabela VII apresentam-se os resultados

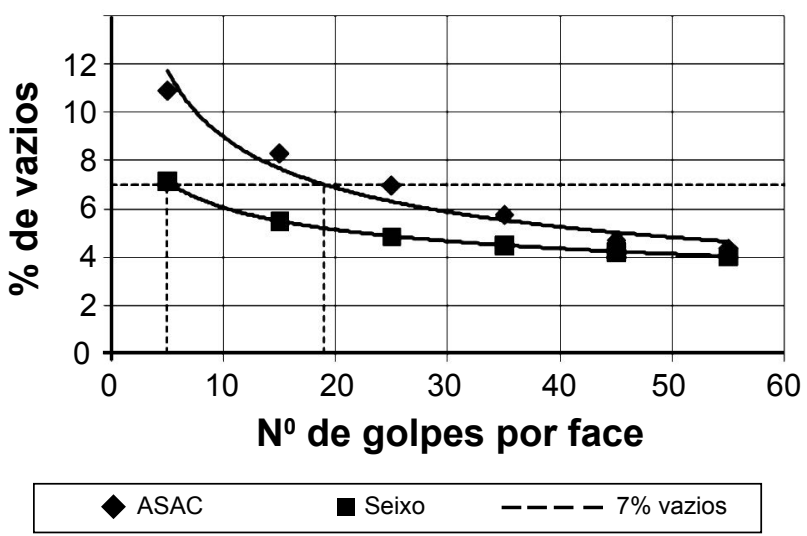

Figura 5: Curvas de compactação das misturas. [Figure 5: Compaction curves of the mixtures.] 


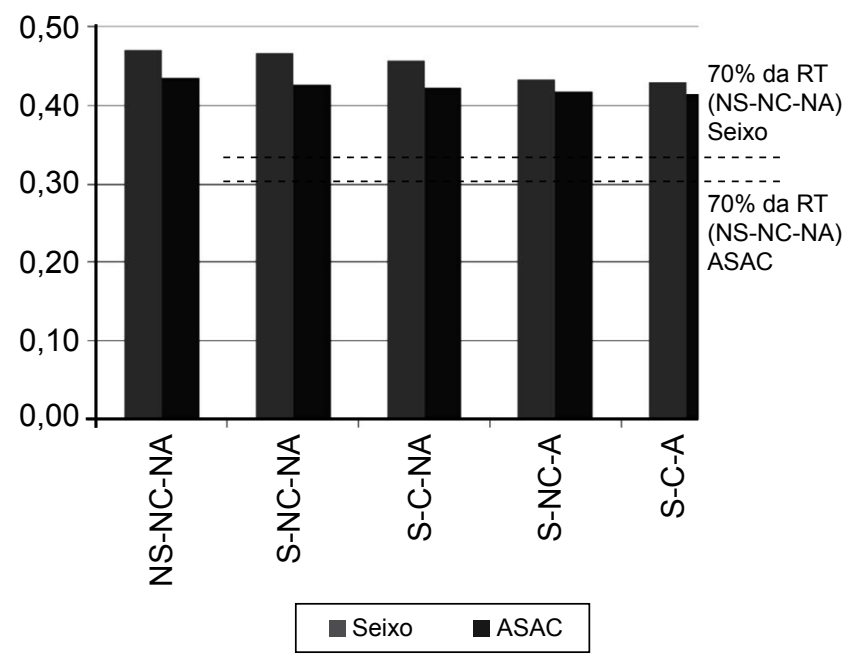

Figura 6: Resultados do ensaio Lottman.

[Figure 6: Results of the Lottman's test.]

do ensaio Lottman para cada tipo de condicionamento: a) não saturado, não congelado e não aquecido, ou seja, sem condicionamento (NS-NC-NA); b) saturado, não congelado e não aquecido (S-NC-NA); c) saturado, congelado e não aquecido (S-C-NA); d) saturado, não congelado e aquecido (S-NC-A); e) saturado, congelado e aquecido (S-C-A).

Analisando-se os dados pode-se afirmar que na condição S-NC-NA, ou seja, para os corpos-de-prova apenas saturados, a mistura com seixo apresentou uma perda de resistência de apenas $1 \%$, enquanto a mistura com ASAC indicou uma redução de sua RT em $2 \%$. Na condição em que os corpos-de-prova foram saturados e congelados (S-CNA), ambas misturas tiveram perdas semelhantes $(3,1 \%$ para a mistura com seixo e $3 \%$ para a mistura com ASAC). No caso do condicionamento com saturação e aquecimento (S-NC-A), as misturas indicaram maior perda de resistências em relação à mistura sem condicionamento, e também respeitante as duas situações anteriores. Observa-se, ainda, que a mistura com seixo proporcionou perda de $8 \%$, ou seja, o dobro do compósito com ASAC que mostrou perda de $4 \%$. Já na condição de completo condicionamento, em que as misturas são saturadas, congeladas e aquecidas (S-C-A), os compósitos com seixo continuaram apresentando redução de resistência, em torno do dobro das misturas com ASAC ( seixo $=8,8 \%$ e ASAC $=4,6 \%$ ). No geral, nota-se que somente nas situações de condicionamento em que ocorre aquecimento, a mistura com seixo mostrou-se mais sensível alusiva à mistura com $\mathrm{ASAC}$, com uma perda de resistência praticamente em dobro. No que tange à operação das vias de Manaus o processo de aquecimento é mais elucidativo que o congelamento, pois as altas temperaturas afetam sobremaneira o comportamento mecânico dos revestimentos locais. Ainda assim, nenhum dos processos de condicionamentos ocasionou RRT sequer próximo do limite aceitável de $70 \%$. Portanto, pode-se afirmar que para as misturas em estudo, o efeito da saturação, congelamento $\mathrm{e}$ aquecimento não comprometeram suas estruturas.

Ensaios uniaxiais de módulo dinâmico - Mistura com ASAC

A Tabela VIII mostra os valores de $\left|E^{*}\right|$ e $\varphi$ para as misturas com ASAC nos ensaios à tração com tensão controlada. A Fig. 7 mostra graficamente esses valores pontuais de $\left|E^{*}\right|$ em função da frequência e da temperatura de ensaio. Determinaram-se os valores de $\left|E^{*}\right|$ e $\varphi$ a partir da média aritmética das leituras de quatro LVDTs conectados ao corpo-de-prova.

Analisando-se a Tabela VIII e a Fig. 7 observa-se que: a) o valor do módulo dinâmico é diretamente proporcional ao aumento da frequência de aplicação de carga e à diminuição da temperatura; b) os valores de ângulo de fase igualmente são dependentes da frequência e da temperatura. Ressaltase que nas temperaturas de $40{ }^{\circ} \mathrm{C} \mathrm{e}-5^{\circ} \mathrm{C}$ registram-se o maior $\left(27,0^{\circ}\right)$ e o menor $\left(4,0^{\circ}\right)$ valor, respectivamente; c) o parâmetro $\left|E^{*}\right|$ nos pontos de ensaio mais extremos, resultaram a $-5^{\circ} \mathrm{C}$ e $25 \mathrm{~Hz}$ um valor de $3503 \mathrm{MPa}$, enquanto a $40{ }^{\circ} \mathrm{C}$ e $0,01 \mathrm{~Hz}$ um módulo igual a $250 \mathrm{MPa}$, ou seja, uma variação de aproximadamente 14 vezes.

Posterior à determinação das curvas módulo dinâmico $\times$ frequência para cada temperatura de ensaio $\left(40{ }^{\circ} \mathrm{C}, 10{ }^{\circ} \mathrm{C}\right.$ e $-5^{\circ} \mathrm{C}$ ), calcularam-se os fatores de translação horizontal $\left(\mathrm{a}_{\mathrm{T}}\right)$ (Tabela IX), resultando na curva mestra da mistura com ASAC, segundo os ensaios à tração com tensão controlada

Tabela VII - Resultados do ensaio Lottman.

[Table VII - Results of the Lottman's test.]

\begin{tabular}{ccccccc}
\hline & \multicolumn{3}{c}{ Seixo } & \multicolumn{3}{c}{ RCD } \\
\cline { 2 - 7 } $\begin{array}{c}\text { Condição de } \\
\text { Ensaio }\end{array}$ & $\begin{array}{c}\text { RT } \\
(\mathrm{MPa})\end{array}$ & $\begin{array}{c}\text { Desvio } \\
\text { Padrão } \\
(\mathrm{MPa})\end{array}$ & $\begin{array}{c}\text { RRT em relação a } \\
\text { NS-NC-NA } \\
(\%)\end{array}$ & $\begin{array}{c}\text { RT } \\
(\mathrm{MPa})\end{array}$ & $\begin{array}{c}\text { Desvio } \\
\text { Padrão } \\
(\mathrm{MPa})\end{array}$ & $\begin{array}{c}\text { RRT em relação a } \\
\text { NS-NC-NA } \\
(\%)\end{array}$ \\
NS-NC-NA & 0,470 & 0,016 & 100,0 & 0,434 & 0,007 & 100,0 \\
S-NC-NA & 0,466 & 0,002 & 99,0 & 0,425 & 0,014 & 98,0 \\
S-C-NA & 0,456 & 0,002 & 96,9 & 0,421 & 0,026 & 97,0 \\
S-NC-A & 0,433 & 0,026 & 92,0 & 0,417 & 0,045 & 96,0 \\
S-C-A & 0,429 & 0,083 & 91,2 & 0,414 & 0,020 & 95,4 \\
\hline
\end{tabular}


Tabela VIII - Resultados dos ensaios uniaxiais de módulo dinâmico, mistura com ASAC.

[Table VIII - Results of the uniaxial dynamic module, mixture with SCCA.]

\begin{tabular}{cccc}
\hline $\begin{array}{c}\text { Temperatura } \\
(\mathrm{T})\end{array}$ & $\begin{array}{c}\text { Frequencia } \\
(\omega), \mathrm{Hz}\end{array}$ & $\begin{array}{c}\text { Módulo dinâmico } \\
\left(\left|E^{*}\right|\right), \mathrm{MPa}\end{array}$ & $\begin{array}{c}\text { Ângulo de Fase } \\
(\varphi), \text { graus }\end{array}$ \\
\hline & 0,01 & 250 & 19,6 \\
& 0,05 & 311 & 18,9 \\
$40{ }^{\circ} \mathrm{C}$ & 0,10 & 354 & 23,4 \\
& 0,50 & 501 & 27,0 \\
& 1,00 & 558 & 22,8 \\
& 5,00 & 752 & 18,0 \\
& 10,00 & 828 & 14,4 \\
& 25,00 & 941 & 18,0 \\
\hline & 0,01 & 1049 & 12,6 \\
& 0,05 & 1214 & 10,8 \\
& 0,10 & 1311 & 7,2 \\
& 0,50 & 1630 & 9,0 \\
& 1,00 & 1705 & 15,6 \\
& 5,00 & 1980 & 9,0 \\
& 10,00 & 2106 & 12,0 \\
& 25,00 & 2295 & 18,0 \\
\hline & 0,01 & 2085 & 4,0 \\
$-5{ }^{\circ} \mathrm{C}$ & 0,05 & 2324 & 7,8 \\
& 0,10 & 2416 & 5,0 \\
& 0,50 & 2653 & 9,0 \\
& 1,00 & 2776 & 11,3 \\
& 5,00 & 3143 & 11,3 \\
& 10,00 & 3307 & 14,4 \\
& 25,00 & 3503 & 18,0 \\
\hline
\end{tabular}

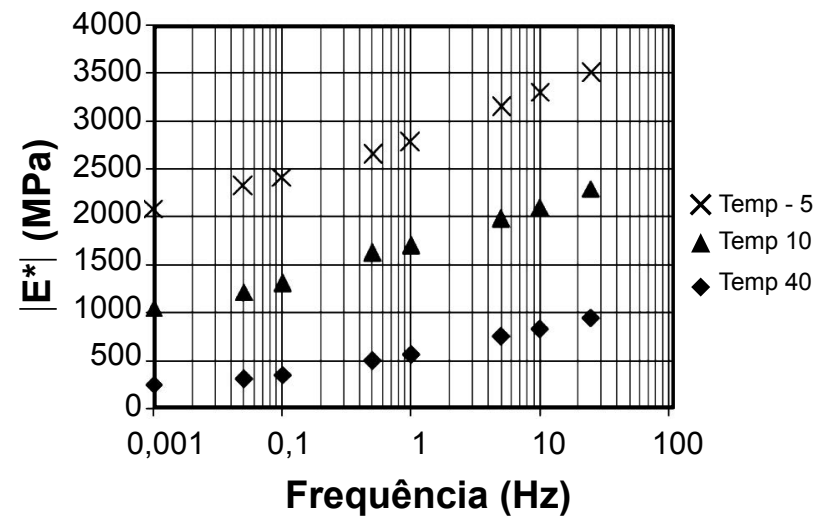

Figura 7: Módulo dinâmico, mistura com ASAC.

[Figure 7: Dynamic module, mixture with SCCA.]

Tabela IX - Fator de translação horizontal, mistura com ASAC.

[Table IX - Shift factor, mixture with SCCA.]

\begin{tabular}{cc}
\hline Temperatura $(\mathrm{T})$ & shift factor $\left(\mathrm{a}_{\mathrm{T}}\right)$ \\
\hline $40{ }^{\circ} \mathrm{C}$ & 0,0002 \\
$25^{\circ} \mathrm{C}$ & 0,02 \\
$10^{\circ} \mathrm{C}$ & 1 \\
$-5^{\circ} \mathrm{C}$ & 700 \\
\hline
\end{tabular}

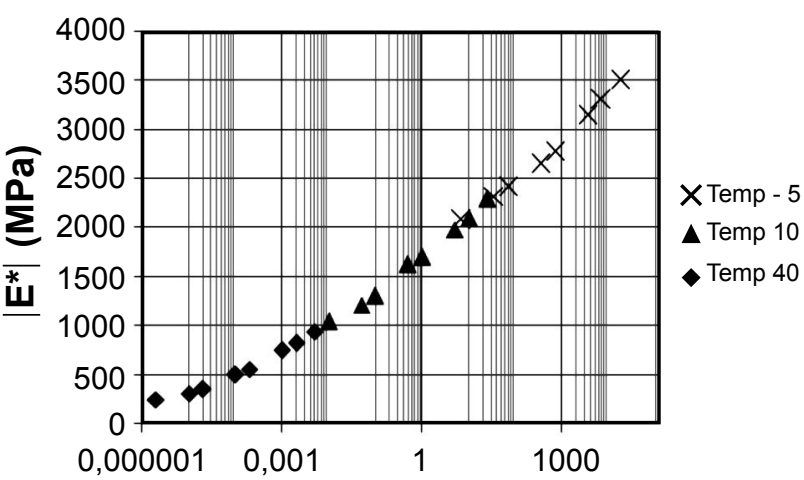

Frequência reduzida $(\mathrm{Hz})$

Figura 8: Curva mestra para o módulo dinâmico, mistura com ASAC.

[Figure 8: Master curve for the dynamic module, mixture with SCCA.]

(Fig. 8). Pela Tabela IX e Fig. 8 nota-se: a) valores de $\left|E^{*}\right|$, às freqüências $(\tilde{\omega}), 700$ vezes superiores ou 5000 vezes inferiores às frequências de ensaio $(\omega)$. Destaca-se a impossibilidade da determinação experimental de tais valores por limitação de equipamentos. 
Ensaios uniaxiais de módulo dinâmico - Mistura com seixo

Na Tabela X e Fig. 9 visualizam-se, respectivamente, para os compósitos com seixo nos ensaios à tração com tensão controlada, os resultados de $\left|E^{*}\right|$ e de $\varphi$, bem como os valores pontuais do $\left|E^{*}\right|$ em função da frequência e da temperatura de ensaio. Examinando-se os dados constantes da citada tabela e figura tem-se que: a) os valores de $\varphi$ variaram entre 14,4 e 38,7 graus; e b) nos pontos mais extremos, tem-se a $-5{ }^{\circ} \mathrm{C}$ e $25 \mathrm{~Hz}$ valor de $\left|E^{*}\right|$ igual a $23621 \mathrm{MPa}$, porem a $35^{\circ} \mathrm{C}$ e $0,01 \mathrm{~Hz}$ resultam $\left|E^{*}\right|$ igual a $81 \mathrm{MPa}$.

Calculando-se os fatores de translação horizontal $\left(\mathrm{a}_{\mathrm{T}}\right)$ constantes na Tabela XI, construiu-se a curva mestra dessa mistura, mostrada na Fig. 10, onde se constata que os fatores de translação horizontal utilizados proporcionaram valores de $\left|E^{*}\right|$, a freqüências ( $\left.\tilde{\omega}\right) 400$ vezes superiores ou aproximadamente 3300 vezes inferiores às frequências de ensaio $(\omega)$. Com a curva mestra construída o valor do módulo dinâmico deixa de ser dependente da frequência e temperatura para ser função exclusivamente da frequência reduzida.

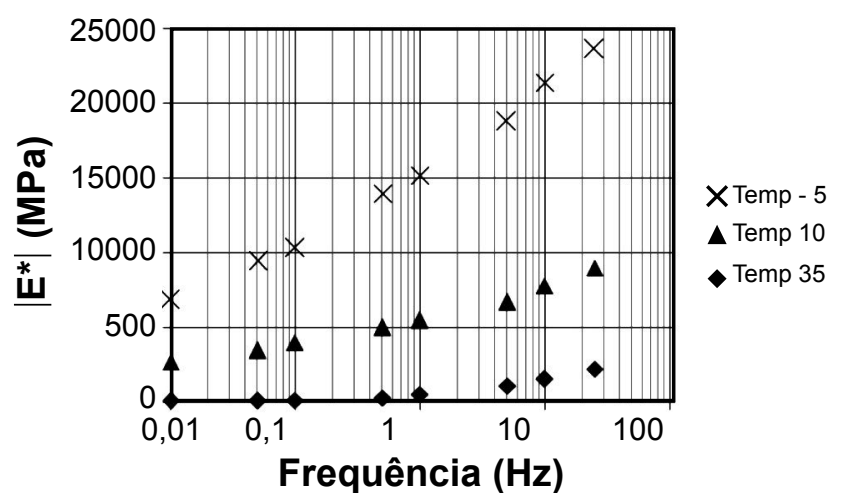

Figura 9: Módulo dinâmico da mistura com seixo. [Figure 9: Dynamic module, mixture with pebble.]

Comparação dos resultados do módulo dinâmico entre as misturas com ASAC e com seixo

A Tabela XII, assim como as Figs. 11 e 12, unifica e exibe em paralelo todos os valores do módulo dinâmico para as misturas com ASAC e com seixo. Neste ponto ressaltam-

Tabela X - Resultados dos ensaios uniaxiais de módulo dinâmico, mistura com seixo.

[Table X-Results of the uniaxial dynamic module, mixture with pebble.]

\begin{tabular}{|c|c|c|c|}
\hline $\begin{array}{l}\text { Temperatura } \\
\text { (T) }\end{array}$ & $\begin{array}{l}\text { Frequência } \\
\qquad(\omega), \mathrm{Hz}\end{array}$ & $\begin{array}{l}\text { Módulo dinâmico } \\
\left(\left|E^{*}\right|\right), \mathrm{MPa}\end{array}$ & $\begin{array}{l}\text { Ângulo de Fase } \\
(\varphi) \text {, graus }\end{array}$ \\
\hline \multirow{8}{*}{$35^{\circ} \mathrm{C}$} & 0,01 & 81 & 38,7 \\
\hline & 0,05 & 103 & 36,0 \\
\hline & 0,10 & 141 & 38,4 \\
\hline & 0,50 & 315 & 36,0 \\
\hline & 1,00 & 554 & 24,6 \\
\hline & 5,00 & 1102 & 15,8 \\
\hline & 10,00 & 1554 & 21,6 \\
\hline & 25,00 & 2251 & 27,0 \\
\hline \multirow{8}{*}{$10^{\circ} \mathrm{C}$} & 0,01 & 2724 & 23,4 \\
\hline & 0,05 & 3501 & 18,9 \\
\hline & 0,10 & 4026 & 26,1 \\
\hline & 0,50 & 5010 & 31,5 \\
\hline & 1,00 & 5490 & 19,8 \\
\hline & 5,00 & 6697 & 15,0 \\
\hline & 10,00 & 7802 & 25,2 \\
\hline & 25,00 & 9022 & 30,0 \\
\hline \multirow{8}{*}{$-5^{\circ} \mathrm{C}$} & 0,01 & 6828 & 18,7 \\
\hline & 0,05 & 9433 & 16,8 \\
\hline & 0,10 & 10285 & 21,6 \\
\hline & 0,50 & 13980 & 21,0 \\
\hline & 1,00 & 15165 & 19,8 \\
\hline & 5,00 & 18777 & 15,0 \\
\hline & 10,00 & 21396 & 14,4 \\
\hline & 25,00 & 23621 & 18,0 \\
\hline
\end{tabular}




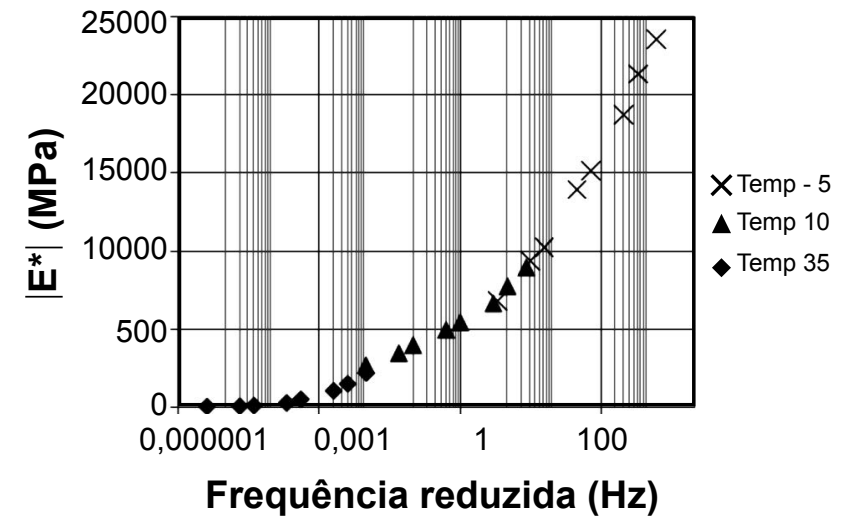

Figura 10: Curva mestra para o módulo dinâmico, mistura com seixo.

[Figure 10: Master curve for the dynamic module, mixture with pebble.]

se: a) na temperatura de $40{ }^{\circ} \mathrm{C}$ e freqüência de $1,0 \mathrm{~Hz}$, notase que os valores do $\left|E^{*}\right|$ das misturas com ASAC e com seixo rolado foram, respectivamente, $558 \mathrm{MPa}$ e $554 \mathrm{MPa}$, ou seja, praticamente iguais. No caso da região de Manaus vale destacar que os pavimentos comumente permanecem
Tabela XI - Fator de translação horizontal, módulo dinâmico da mistura com seixo.

[Table IX - Shift factor, mixture with pebble.]

\begin{tabular}{cc}
\hline Temperatura $(\mathrm{T})$ & Shift factor $\left(\mathrm{a}_{\mathrm{T}}\right)$ \\
\hline $35^{\circ} \mathrm{C}$ & 0,0003 \\
$10{ }^{\circ} \mathrm{C}$ & 1 \\
$-5^{\circ} \mathrm{C}$ & 400 \\
\hline
\end{tabular}

a maior parte do dia submetidos a temperaturas geralmente superiores a $40{ }^{\circ} \mathrm{C}$; b) conforme diminui-se a temperatura de ensaio e/ou aumenta-se a frequência de aplicação de carga, a mistura com seixo vai adquirindo uma rigidez muito superior às misturas com ASAC. Tomando-se como exemplo a menor temperatura e maior frequência ensaiada $\left(-5^{\circ} \mathrm{C}\right.$ e $25,0 \mathrm{~Hz}$ ), observa-se que a mistura com seixo apresentou valor de $\left|E^{*}\right|$ igual a $23621 \mathrm{MPa}$ enquanto o compósito com ASAC indicou o valor de $\left|E^{*}\right|$ igual a $3503 \mathrm{MPa}$, ou seja, a mistura com seixo mostrou-se cerca de 6,7 vezes mais rígida relativa às mistura com ASAC, assim sendo, mais susceptível a trincamento por fadiga; c) por outro

Tabela XII - Resultados dos ensaios uniaxiais de módulo dinâmico das misturas com ASAC e seixo. [Table XII - Results of the uniaxial dynamic modulus of the mixtures with SCCA and pebble.]

\begin{tabular}{|c|c|c|c|}
\hline Temperatura & Frequência, $\mathrm{Hz}$ & $\begin{array}{c}\text { Módulo dinâmico seixo } \\
\left(\left|\mathrm{E}^{*}\right|\right), \mathrm{MPa}\end{array}$ & $\begin{array}{l}\text { Módulo dinâmico ASAC } \\
\left(\left|E^{*}\right|\right), \mathrm{MPa}\end{array}$ \\
\hline \multirow{8}{*}{$\begin{array}{c}40^{\circ} \mathrm{C}(\text { ASAC) e } \\
35^{\circ} \mathrm{C} \text { (seixo) }\end{array}$} & 0,01 & 81 & 250 \\
\hline & 0,05 & 103 & 311 \\
\hline & 0,10 & 141 & 354 \\
\hline & 0,50 & 315 & 501 \\
\hline & 1,00 & 554 & 558 \\
\hline & 5,00 & 1102 & 752 \\
\hline & 10,00 & 1554 & 828 \\
\hline & 25,00 & 2251 & 941 \\
\hline \multirow{8}{*}{$10^{\circ} \mathrm{C}$} & 0,01 & 2724 & 1049 \\
\hline & 0,05 & 3501 & 1214 \\
\hline & 0,10 & 4026 & 1311 \\
\hline & 0,50 & 5010 & 1630 \\
\hline & 1,00 & 5490 & 1705 \\
\hline & 5,00 & 6697 & 1980 \\
\hline & 10,00 & 7802 & 2106 \\
\hline & 25,00 & 9022 & 2295 \\
\hline \multirow{8}{*}{$-5^{\circ} \mathrm{C}$} & 0,01 & 6828 & 2085 \\
\hline & 0,05 & 9433 & 2324 \\
\hline & 0,10 & 10285 & 2416 \\
\hline & 0,50 & 13980 & 2653 \\
\hline & 1,00 & 15165 & 2776 \\
\hline & 5,00 & 18777 & 3143 \\
\hline & 10,00 & 21396 & 3307 \\
\hline & 25,00 & 23621 & 3503 \\
\hline
\end{tabular}




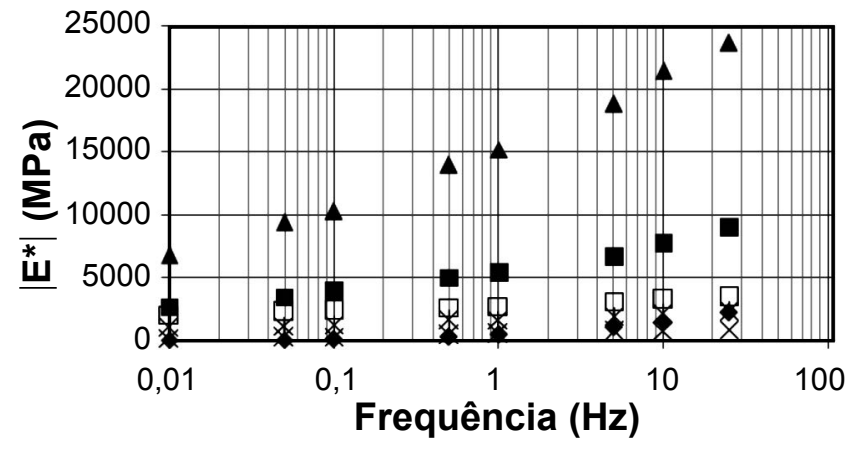

- SEIXO TC - Temp 35 SEIXO TC - Temp $10 \quad \boldsymbol{\Delta}$ SEIXO TC - Temp -5 X ASAC TC - Temp $40 \quad$ * ASAC TC - Temp $10 \quad \square$ ASAC TC - Temp -5

Figura 11: Módulo dinâmico $\times$ frequências das misturas com ASAC e seixo.

[Figure 11: Dynamic module $\times$ frequency of the mixtures with SCCA and pebble.]

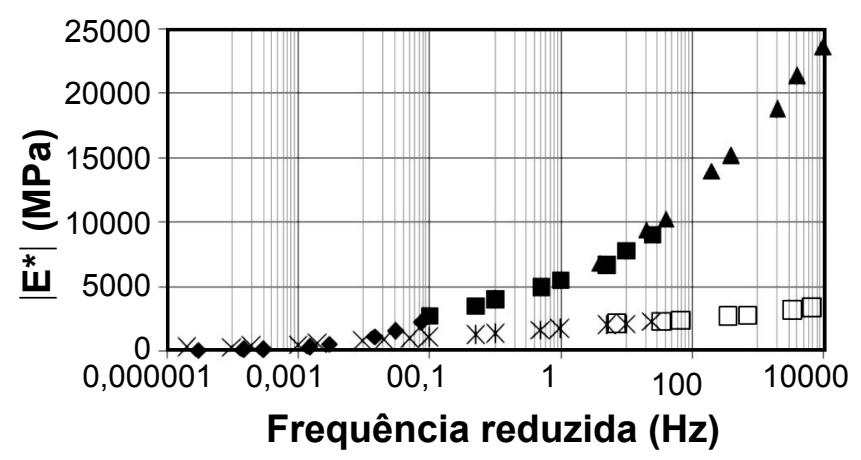

- SEIXO TC - Temp 35 SEIXO TC - Temp $10 \quad \Delta$ SEIXO TC - Temp -5 $\times$ ASAC TC - Temp $40 \quad$ * ASAC TC - Temp $10 \quad \square$ ASAC TC - Temp -5

Figura 12: Curvas mestras dos ensaios uniaxiais de módulo dinâmico das misturas com ASAC e seixo.

[Figure 12: Master curves of the uniaxial dynamic modulus of the mixtures with SCCA and pebble.]

lado, tomando-se como exemplo a mais alta temperatura e menor frequência ensaiadas $\left(40{ }^{\circ} \mathrm{C}\right.$ e $\left.0,01 \mathrm{~Hz}\right)$ obteve-se para a mistura com ASAC valor de $\left|E^{*}\right|$ igual a $250 \mathrm{MPa}$ e para o compósito com seixo $\left|E^{*}\right|$ resultou em $81 \mathrm{MPa}$. Nesta situação a mistura com ASAC mostrou-se aproximadamente três vezes mais rígida comparada à mistura com seixo, logo menos susceptível às deformações permanentes, como, por exemplo, afundamento de trilhas de roda.

\section{CONCLUSÕES}

A areia artificial (areia ASAC) como material alternativo, mostrou desempenho mecânico satisfatório, proporcionando melhor aproveitamento do material britado para obtenção dos agregados sintéticos (ASAC) e minimização da extração do recurso natural.Os ensaios de verificação da potencialidade à calcinação da Amostra SUC, nas condições in natura e calcinada, indicaram o não atendimento às especificações DNIT, para uso como ASAC, particularmente quanto ao
Abrasão Los Angeles, onde o desgaste foi de $70 \%$, assim como quanto aos intervalos de óxidos recomendados pelo DNER $\left(\mathrm{SiO}_{2}\right.$ e $\left.\mathrm{Al}_{2} \mathrm{O}_{3}\right)$. A caracterização do agregado ASAC confirmou que são materiais leves (Wur $=1062,00 \mathrm{~kg} / \mathrm{m}^{3}$ ) com alto poder de absorção, levando a um maior consumo de ligante na composição da mistura. A dosagem mostrou um teor de projeto no valor de 9,40\% para a mistura com ASAC, onde também participou em sua composição areia ASAC, e igual a $4,5 \%$ para o compósito com seixo e areia natural, ou seja, a mistura com o material alternativo consumiu praticamente o dobro de ligante relativo ao compósito com o material aluvionar. Por outro lado, o consumo de cimento da mistura com ASAC foi de 1,4\% respeitante ao resultado igual a $3,1 \%$ do compósito com seixo. A caracterização do CAP 50/70, conforme critério Superpave, mostrou sua temperatura de serviço não superior a $64{ }^{\circ} \mathrm{C}$. Tal resultado sinaliza que o material é adequado para Manaus, onde suas vias estão submetidas, em geral, à temperatura superficial superior a $50{ }^{\circ} \mathrm{C}$ nos períodos mais quentes do ano. Os ensaios de perda de resistência por umidade induzida (Lottman) indicaram que nas situações de condicionamento mais elucidativas, particularmente no que tange à operação das vias de Manaus, ou seja, nas situações onde ocorreu o aquecimento, a mistura com seixo rolado mostrou-se mais susceptível comparada à mistura com ASAC, com uma perda de resistência praticamente em dobro. Ainda assim, nenhum dos compósitos mostrou sua estrutura comprometida pelo efeito dos processos de condicionamentos (saturação, congelamento e aquecimento). A suavização e regressão dos dados experimentais possibilitou a completa eliminação dos ruídos elétricos, definindo de forma mais nítida os picos de tensão e deformação, bem como facilitou a obtenção dos valores do $\left|E^{*}\right|$ e $\varphi$. Os resultados do módulo dinâmico uniaxial para ambas as misturas e na condição de ensaio (tração com tensão controlada), corroborou os estudos teóricos, ou seja, o parâmetro $\left|E^{*}\right|$ (materiais viscoelásticos) é diretamente proporcional ao aumento da frequência de aplicação de carga e à diminuição da temperatura. A construção das curvas mestras, para o conjunto dos compósitos (ASAC e seixo), proporcionou determinar, a baixas e altas frequências, os valores de $\left|E^{*}\right|$. Tais valores seriam impraticáveis de serem obtidos experimentalmente, por limitação tecnológica dos equipamentos ora existentes. Os módulos dinâmicos das misturas com ASAC apresentaram-se superiores aos das misturas com seixo para altas temperaturas de ensaio e baixas frequências de aplicação de carga (condições que retratam a situação a que são submetidos os pavimentos locais), demonstrando sua menor suscetibilidade a deformações permanentes. No caso de baixas temperaturas e altas frequências, o processo se inverteu e as misturas com seixo passaram a apresentar maiores valores de $\left|E^{*}\right|$ frente os compósitos com ASAC, logo mais suscetíveis a trincamento por fadiga em razão da maior rigidez. As misturas com o material alternativo (ASAC) mostraram-se menos susceptíveis às variações de temperatura, indicativo aos compósitos com seixo, fato este observado pela menor variação dos valores do parâmetro 
$\left|E^{*}\right|$ e, consequentemente, sua rigidez. Em resumo, pode-se concluir que: a) os agregados sinterizados de argila calcinada (ASAC) substituem com eficácia os agregados naturais e finitos (seixo) na composição das misturas asfálticas, melhorando seu desempenho em campo e contribuindo para minimizar o grave problema da escassez de matériaprima para obtenção do agregado pétreo na Amazônia; e b) a determinação do módulo dinâmico proporciona a obtenção de uma propriedade viscoelástica mais próxima da realidade em campo.

\section{REFERÊNCIAS}

[1] Detran-AM - Departamento Estadual de Trânsito do Amazonas, "Frota de veículos" Manaus, AM (2010).

[2] A. Sides, J. Uzan, M. Perl, ASTM J. Testing Evaluation 59 (1985).

[3] C. A. Frota, C. L. S. Alencar, C. P. L. Silva, Anais $35^{\text {a }}$ Reunião Anual de Pavimentação, ABPv, Rio de Janeiro, RJ (2004).

[4] C. A. Frota, C. L. Silva, F. R. G. Nunes, Anais V Jornadas Luso-Brasileiras de Pavimentos, Recife, PE (2006).

[5] C. A. Frota, C. L. Silva, M. G. R. Santos, R. V. P Silva, Anais $35^{\text {a }}$ Reunião Anual de Pavimentação, Rio de Janeiro, RJ, (2004).

[6] C. A. Frota, D. M. Melo, C. L. Silva, E M. G. R. Santos, Anais VI Jornadas Luso-Brasileiras de Pavimentos, Porto, Portugal (2007).

[7] C. A. Frota, F. R. G. Nunes, C. L. Silva, $12^{\text {a }}$ Reunião de Pavimentação Urbana, Aracaju, SE (2003).

[8] C. A. Frota, F. R. G. Nunes, C. L. Silva, D. M. Melo, M. G. R. Santos, Cerâmica 53, 327 (2007) 255.

[9] F. R. G. Nunes, Diss. Mestrado, Programa de Mestrado em Engenharia de Transportes, Universidade Federal do Ceará, Fortaleza, CE (2006).

[10] M. G. R. Santos, Diss. Mestrado, Programa de Mestrado em Geotecnia, Universidade de Brasília - UnB, Brasília, DF (2007).

[11] C. L. Silva, F. R. G. Nunes, C. A. Frota, $15^{a}$ Reunião de Pavimentação Urbana, Salvador, BA (2008).

[12] C. L. Silva, C. A. Frota, Cerâmica, 59, 352 (2013) 508.

[13] F. G. S. Batista, Diss. Mestrado, Instituto Militar de Engenharia, Rio de Janeiro, RJ (2004).

[14] G. L. L. Cabral, Diss. Mestrado, Instituto Militar de Engenharia, Rio de Janeiro, RJ (2005).

[15] R. R. Nascimento, Diss. Mestrado, Coordenação dos Programas de Pós-Graduação de Engenharia, Universidade Federal do Rio de Janeiro (COPPE-UFRJ), Rio de Janeiro, RJ (2004).

[16] M. A. V. Silva, Diss. Mestrado, Instituto Militar de Engenharia, Rio de Janeiro, RJ (2006).

[17] R. A. Santos, Diss. Mestrado, Instituto Militar de Engenharia, Rio de Janeiro, RJ (2008).

[18] G. L. L. Cabral, Tese Dr., Coordenação dos Programas de Pós-Graduação de Engenharia, Universidade Federal do Rio de Janeiro (COPPE-UFRJ), Rio de Janeiro, RJ (2011).

[19] ABNT - Associação Brasileira de Normas Técnicas,
“NBR 7181 - Solo: Análise granulométrica,” Rio de Janeiro, (1984).

[20] ABNT - Associação Brasileira de Normas Técnicas, "NBR 6459 - Solo: Determinação do limite de liquidez," Rio de Janeiro (1984).

[21] ABNT - Associação Brasileira de Normas Técnicas, "NBR 7180 - Solo: Determinação do limite de plasticidade," Rio de Janeiro (1984).

[22] DNER - Departamento Nacional de Infra-Estrutura de Transportes, "ME 223/94: Argilas para a fabricação de agregados sintéticos de argila calcinada: Seleção expedita pelo Processo de Fervura", Rio de Janeiro (1994).

[23] DNER - Departamento Nacional de Infra-Estrutura de Transportes, "ME 225/94: Agregado sintético de argila calcinada: Determinação da Perda de Massa após Fervura", Rio de Janeiro (1994).

[24] DNER - Departamento Nacional de Infra-Estrutura de Transportes, "ME 222/94: Agregado sintético fabricado com argila: Desgaste por Abrasão", Rio de Janeiro (1994).

[25] ASTM - American Society for Testing and Materials, "C 136-95: Standard Test Method for Sieve Analysis of Fine and Coarse Aggregates", USA (1995).

[26] ASTM - American Society for Testing and Materials, "ASTM C 127-88: Standard Test Method for Specific Gravity and Absorção of Coarse Aggregate", USA (1988).

[27] AASHTO - American Association of State Highway and Transportation Officials, "AASHTO T 19-93: Standard Test Method for Unit Weight and Voids in Aggregate", USA (1997).

[28] ASTM - American Society for Testing and Materials, "C 131-96: Standard Test Method for Resistance to Degradation of Small-Size Coarse Aggregate by Abrasion and Impact in the Los Angeles Machine", USA (1996).

[29] DNER - Departamento Nacional de Infra-Estrutura de Transportes, "ME 078/94: Agregado graúdo: adesividade a ligante betuminoso", Rio de Janeiro (1994).

[30] ASTM - American Society for Testing and Materials, "C 128-93: Standard Test Method for Specific Gravity and Absorção of Fine Aggregate", USA (1988).

[31] AASHTO - American Association of State Highway and Transportation Officials, "AASHTO T 19-93: Standard Test Method for Unit Weight and Voids in Aggregate", USA (1997).

[32] SHRP - "Superior Performance Asphalt Pavements (Superpave), The Product of SHRP Asphalt Research Program, Superpave Series $N^{\circ}$. 1", Strategic Highway Research Program (1994).

[33] SHRP - "Superior Performing Asphalt Pavements (Superpave): The Product of SHRP Asphalt Research Program, Superpave Series $N^{\circ}$. 2", Strategic Highway Research Program (1994).

[34] DNER - Departamento Nacional de Infra-Estrutura de Transportes, "DNER ME 085/94: Material finamente pulverizado: Determinação da massa específica real", Rio de Janeiro (1994).

[35] DNER - Departamento Nacional de Infra-Estrutura de Transportes, "EM 367/97: Material de enchimento para 
misturas betuminosas", Rio de Janeiro (1997).

[36] TRB - "Bailey Method for Gradation Selection in HotMix Asphalt Mixture Design", Transportation Research Board of the National Academies, Vols. E-CIRCULAR 044, Washington, DC (2002).

[37] DNER - Departamento Nacional de Infra-Estrutura de Transportes, "DNER ME 043/95: Misturas betuminosas a quente - Ensaio Marshall", Rio de Janeiro (1995).

[38] AASHTO - American Association of State Highway and Transportation Officials, "PP-2 - Standard Pratice for Mixture Conditioning of Mix Asphalt", Washington, DC (1999).

[39] DNER - Departamento Nacional de Infra-Estrutura de Transportes, "ME 004/94: Material Betuminoso: determinação da viscosidade Saybolt-Furol a alta temperatura método da película delgada", Rio de Janeiro (1994).
[40] ASTM - American Society for Testing and Materials, "D 2041-00: Standard Test Method for Theoretical Maximum Specific Gravity and Density of Bituminous Paving Mixtures", USA (2000).

[41] AASHTO - American Association of State Highway and Transportation Officials, “AASHTO T 283-03: Resistence of Compacted Asphalt Mixtures to Moisture-Induced Damage", USA (2003).

[42] ABNT - Associação Brasileira de Normas Técnicas, "NBR 6502: Rochas e Solos", Rio de Janeiro (1995).

[43] DNER - Departamento Nacional de Infra-Estrutura de Transportes, "EM 230/94: Agregados Sintéticos Graúdos de Argila Calcinada", Rio de Janeiro (1994).

[44] S. M. Chaar, P. R. C. Couceiro, C. A. Frota, J. S. Chaar, K. S. Souza, C. L. Silva, Painel, Universidade Federal do Amazonas, Manaus, AM (2008).

(Rec. 09/07/2013, Ac. 21/07/2013) 